\title{
Complications of splintage in congenital dislocation of the hip
}

\author{
V G Langkamer, N M P Clarke, P Witherow
}

\begin{abstract}
The use of abduction splintage in the treatment of congenital dislocation of the hip has an important morbidity. Six children who developed complications are presented in this paper. Sustained splintage of an unreduced hip, overcorrection of the femoral head displacement, avascular necrosis of the femoral head, full thickness pressure sores, and excessive tibial torsion may occur as a consequence of treatment. Expert supervision of abduction splintage, correct case selection, and regular review are necessary to reduce the incidence of such complications.
\end{abstract}

The treatment of congenital dislocation of the hip is associated with significant complications. Two factors that may influence their development are the method and supervision of the chosen splintage and the age of the patient at the commencement of treatment. Before the introduction of splintage, the management of congenital dislocation of the hip consisted of either traction $^{1}$ or forceful closed manipulation of the femoral head. ${ }^{2}$ Closed reduction and prolonged fixed splintage in a cast was promoted on the basis that redislocation would be prevented and in some cases even the presence of hypertrophied soft tissue obstructing reduction would be overcome with prolonged pressure. ${ }^{3}$ Sustained immobilisation was associated with redislocation in splintage in $32 \%$ of cases and avascular necrosis of the femoral capital epiphysis in $50 \% .^{45}$

Splints such as the Denis Browne rigid bar abduction device were originally introduced to reduce the total time spent in a cast and were not recommended for use alone. ${ }^{6}$ Full abduction was rigidly maintained for over nine months. Nursing and maintenance of these children was greatly improved by the introduction of lighter, removable splints of which the Von Rosen was one example. This device consisted of malleable aluminium strips covered in washable rubber and applied around the shoulders, trunk, and thighs.

The association between compressive vascular injury of the femoral capital ossific epiphysis and forced abduction of the hip was supported by cadaver angiographic studies demonstrating significant vascular compromise in the 'frog' position commonly used in rigid abduction splints. ${ }^{8}$ Experimental avascular necrosis was reproduced by immobilising the hip joints of immature pigs in plasters in full abduction after artificial adductor shortening. 9 This awareness led to a preference for a splint which utilised hip flexion to site the femoral head and gradual natural abduction to overcome adductor contracture. The most popular dynamic splint remains the Pavlik harness.

The Pavlik harness was introduced in 1944 and consisted of an adjustable circumferential abdominal band with crossed posterior straps and leg stirrups. The straps were adjusted to provide hip flexion and free abduction. Pavlik reported an $84 \%$ success rate with no avascular necrosis. ${ }^{10}$ Suitable modifications in the treatment protocols have produced success rates of 90 to $99 \%$ with no avascular necrosis. ${ }^{11} 12$ However, important complications have also been reported from the widespread use of this harness. Avascular necrosis has been reported to be as high as $28 \% .^{13}{ }^{14}$ Failure to achieve reduction and redislocation ${ }^{12-15}$ as well as nerve and skin complications have been reported. ${ }^{11} 1215$

The decision to apply splintage early or delayed is controversial and may influence the development of complications, especially avascular necrosis. Early splintage was advocated in 1929 by Putti who recommended cushioned abduction as soon as the diagnosis was apparent. ${ }^{16}$ Putti recognised a group which underwent 'spontaneous reduction' and did not require treatment. Early treatment was encouraged in order to avoid the complications of delay, which included difficult reduction and an increase in the incidence of avascular necrosis. ${ }^{11} 121718$ Others have reported avascular necrosis to be higher during treatment in the first six months of life when the developing capital epiphysis is most vulnerable to pressure. ${ }^{17-19}$ Barlow's observation that $60 \%$ of unstable hips would resolve spontaneously further behoves those who choose to splint all unstable hips at birth to ensure that 'normal' hips do not sustain a compressive vascular injury with subsequent proximal femoral growth plate damage and deformity. ${ }^{20}$

\section{Case histories}

CASE 1 (OVERCORRECTION OF FEMORAL HEAD DISPLACEMENT)

A girl delivered normally was found to have an unstable right hip on examination. Splintage was commenced on the fourth day in a Pavlik harness and she was reassessed clinically at intervals of three weeks. Clinical examination at 6 weeks of age did not detect any abnormality but a radiograph suggested that the right hip was positioned wide of the acetabulum. An arthrogram confirmed overcorrection with the development of a large superior pool (fig 1). At 


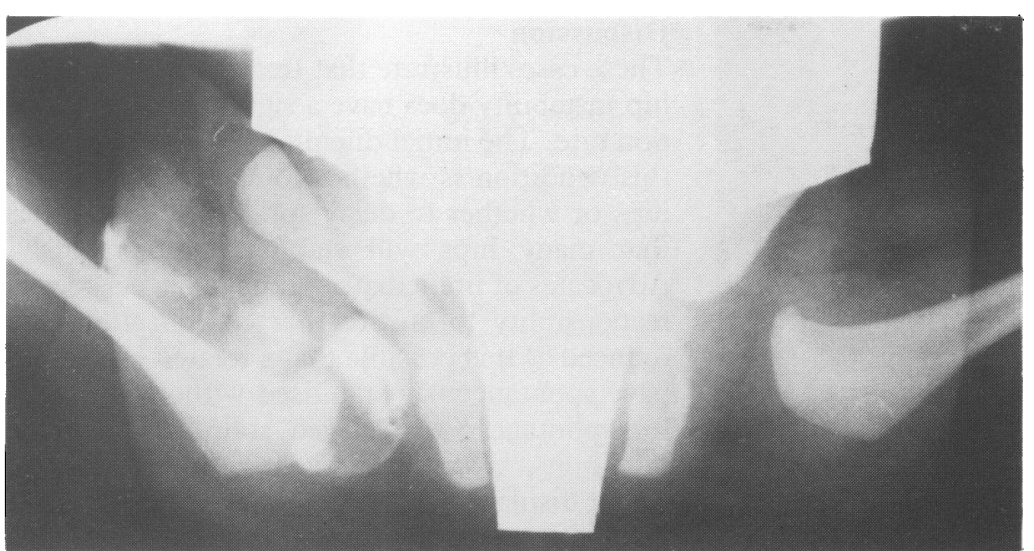

Figure 1 Case 1 (overcorrection of femoral head displacement). Arthrogram of the right hip in a 6 week old girl. Note the inferior position of the overcorrected femoral capital epiphysis and superior pooling of the contrast medium.

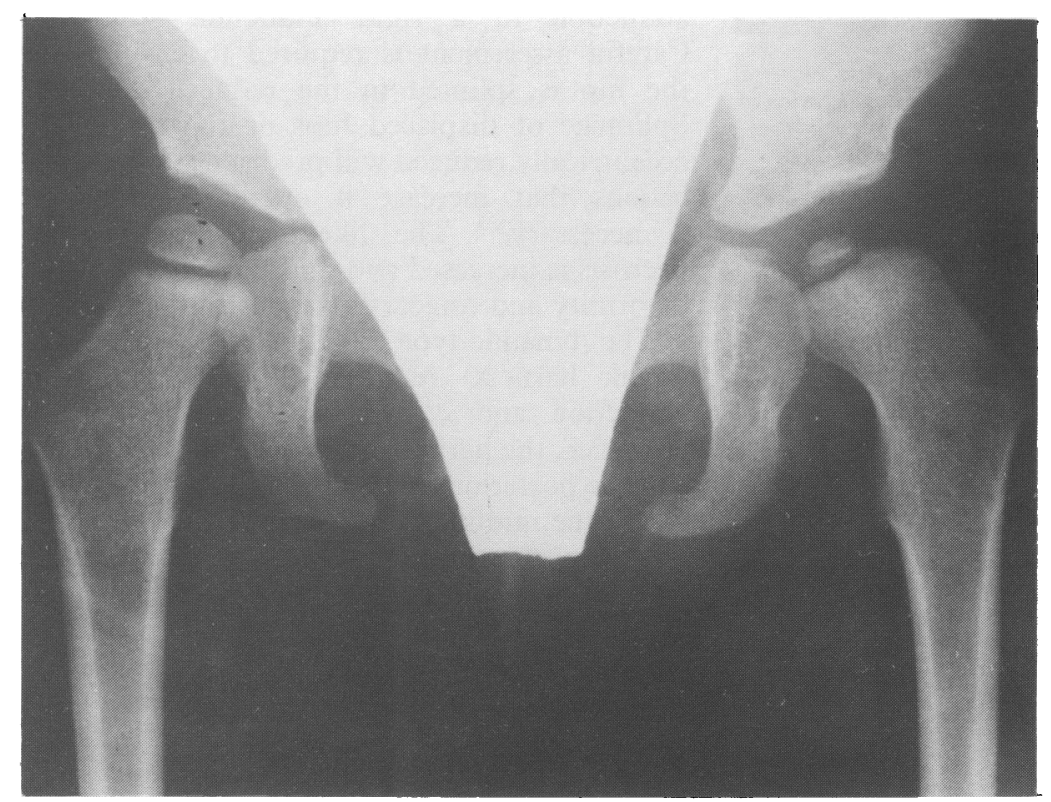

Figure 2 Case 2 (epiphysitis). Pelvic radiograph of a girl aged 2 years. There is a delay in ossification of the left capital femoral ossific nucleus with some fragmentation; this appearance is suggestive of avascular necrosis.

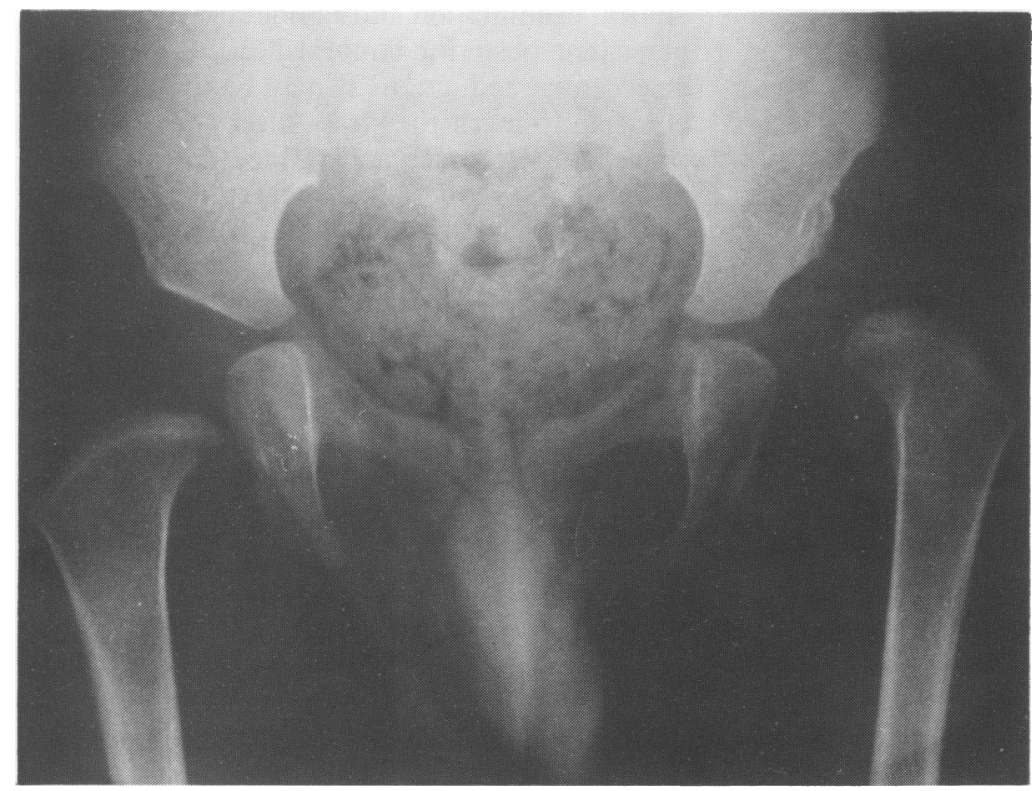

Figure 3 Case 3 (persistent hip dislocation in the splint). Pelvic radiograph of a boy at 9 months of age. This radiograph was taken after 9 months of splintage. The left hip remains dislocated.
7 months an open reduction was performed and the acetabulum was observed to be shallow. Reduction could be achieved only after considerable soft tissue resection and recovery was uneventful after six months of serial plaster spicas.

\section{CASE 2 (EPIPHYSITIS)}

A girl was born prematurely and at birth a dislocated left knee and hip were diagnosed. The knee dislocation was reduced in serial plasters and the hip was treated immediately in a Von Rosen splint. At 2 months of age the splint was causing excessive pressure around the front of the thighs and a Craig splint was applied for a further six months. The left femoral capital epiphysis was considerably delayed in development and at 2 years the radiological signs of a epiphysitis were confirmed (fig 2).

\section{CASE 3 (PERSISTENT HIP DISLOCATION IN THE SPLINT)}

A boy was found to have 'clicking' hips at birth. An Aberdeen type polythene abduction splint was applied for nine months during which time he was reassessed regularly. At 9 months of age the left hip was found to be dislocated (fig 3). At 11 months an open reduction was necessary, but the dislocation persisted. Redislocation continued to occur because of a shallow acetabulum and stability could not be achieved even after a Pemberton pelvic osteotomy (fig 4). Persistent anterior acetabular deficiency required a further acetabuloplasty and a derotation femoral osteotomy.

\section{CASE 4 (FULL THICKNESS PRESSURE SORE)}

A girl delivered normally was diagnosed as having clinical bilateral hip instability at birth. A Von Rosen splint was applied and the parents instructed that it should not be removed until the child was reassessed. Three weeks later a red pressure area was noted over the right iliac crest posteriorly. Although the lesion was dressed and the splint changed to an Aberdeen type polythene abduction splint the lesion ulcerated (fig 5). At three years of age she is clinically normal but has a $2 \mathrm{~cm}$ by $1 \mathrm{~cm}$ full thickness scar at the site of the ulcer.

CASE 5 (FAILURE OF FEMORAL HEAD RELOCATION) A girl was born by caesarean section and diagnosed as having 'clicking' hips at birth. She was treated in double nappies until 6 weeks of age and subsequently a Pavlik harness was applied. The mother was instructed to remove this for bathing. At $\mathbf{4}$ months the harness was clearly too small and incorrectly applied with the abdominal strap below her waist. A radiograph demonstrated that the left hip had not been concentrically reduced. Successful reduction was achieved with a larger, correctly fitted harness. Treatment was continued in this for a further six months. 


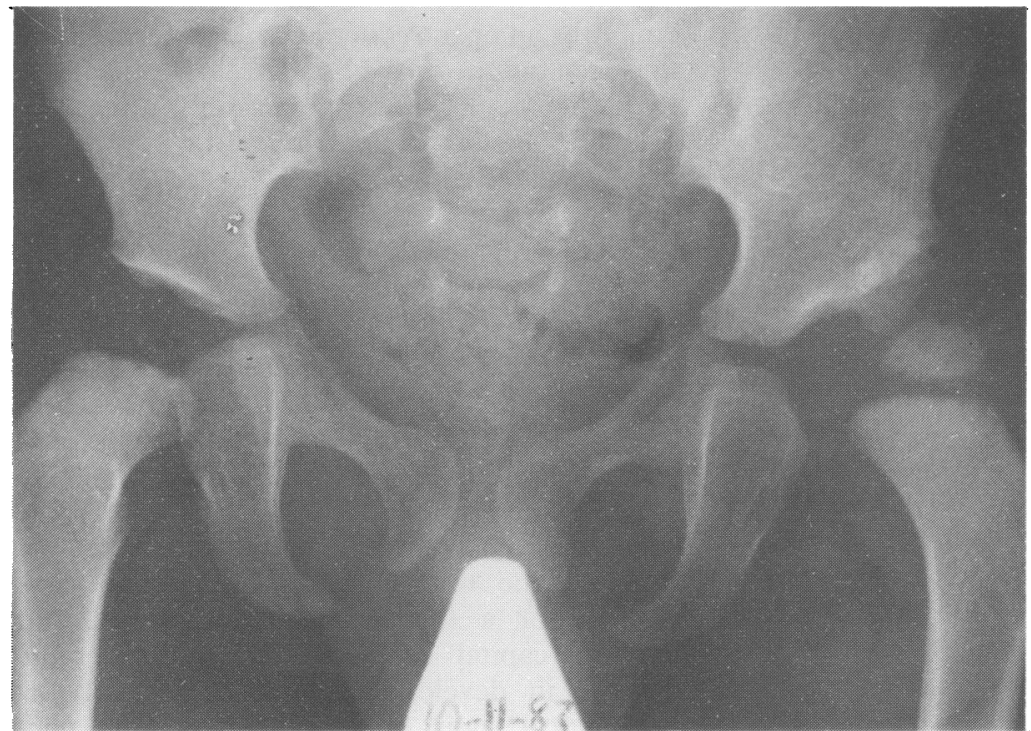

Figure 4 Case 3 (persistent hip dislocation in the splint). Pelvic radiograph of case 3 after a Pemberton pelvis osteotomy. The left hip remains dislocated. Note also delayed ossification of the right femoral capital epiphysis.

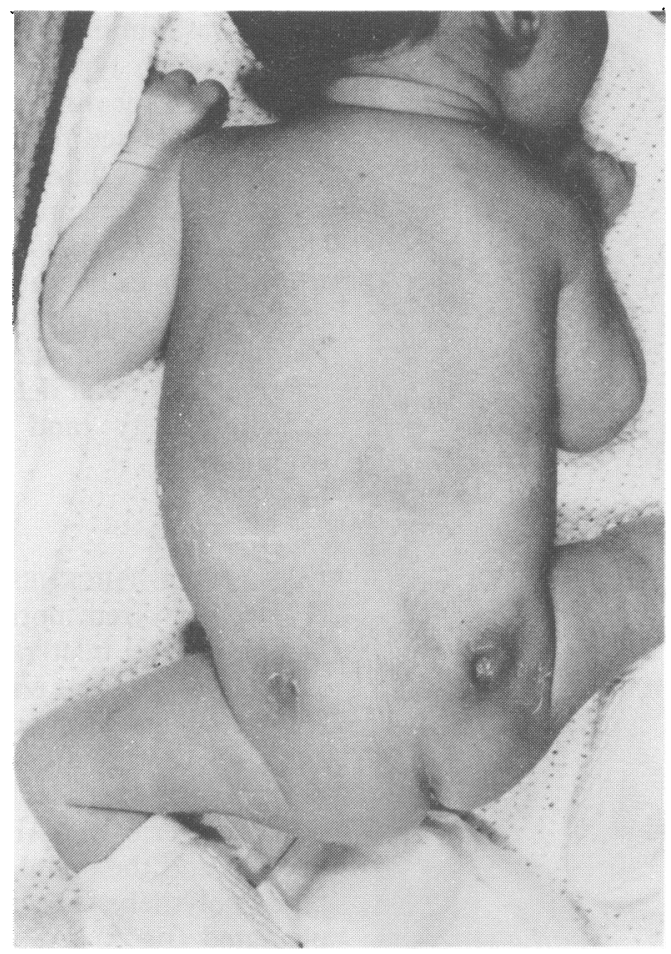

Figure 5 Case 4 (full thickness pressure sores). Girl at 3 weeks of age shortly after the removal of a Von Rosen splint. The full thickness pressure sores seen here eventually healed but large scars remain at 3 years of age.

CASE 6 (EXTERNAL TIBIAL TORSION)

A boy born in another country was noted to have a dislocatable left hip at birth and was treated in a Pavlik harness for six months. At that time the child's father was concerned that an 'ankle strap' which had been added to the splint was exceptionally tight and was turning the left leg out excessively. Splintage was continued for 11 months. At the age of 5 years, there was a 60 degree external rotation deformity of the tibia confirmed by computed tomography. The right leg is normal.

\section{Discussion}

These cases illustrate that treatment of neonatal hip instability does have a significant complication rate. The initial dilemma of those who treat this condition is whether to splint all unstable hips or whether to delay splintage on the basis that many hips will stabilise spontaneously. Advocates of immediate splintage have a special responsiblity to ensure that complications are reduced as it is possible that a treated hip would have spontaneously stabilised without the need for splintage. ${ }^{2021}$ Delayed splintage risks the possibility of clinical examination failing to detect displacement because the clinical signs of instability gradually resolve in the early weeks. This resolution is associated with an increasing limitation of abduction and adductor tightness. This renders the femoral head vulnerable to compressive vascular injury with forced hip abduction in a rigid abduction splint. ${ }^{92}$ Careful assessment is required to ensure that the hip is splinted in the reduced position. Splintage of displaced hips or those that are eccentrically reduced will predispose to complications that increase if operative reduction is necessary. ${ }^{23}$ The likelihood of avascular necrosis is increased and this may predispose to deformity and osteoarthrosis in later years. ${ }^{24}$

The dynamic type of splintage (for example, Pavlik harness) reduces the risk of forced abduction and compressive vascular injury. However, the harness must be applied correctly and the posterior straps used to limit adduction across the midline rather than abduct the hips. Similarly, the position of hip flexion is critical. The hip should be flexed to just above 90 degrees. ${ }^{12}$ Excessive flexion may result in femoral nerve palsy ${ }^{11} 1215$ or inferior displacement of the hip. ${ }^{15}$ Medial knee ligament instability may occur as a consequence of valgus strain applied by a medial strap slipping over the knee. ${ }^{25}$ For these reasons expert supervision of an infant in a harness is required on perhaps a weekly basis. Frequent strap adjustments are required and the position of the hip checked by clinical examination and radiography to prevent persistent posterior femoral head displacement or eccentric reduction. It may be useful for the clinician to mark the straps after adjustment to prevent interference and reduce the likelihood of losing the reduction. If necessary, treatment may have to be abandoned in favour of alternative procedures such as adductor tenotomy or planned open reduction. ${ }^{26-29}$

Ultrasound has proved to be a useful adjunct to regular clinical examination of hips splinted particularly in the Pavlik harness. Where ultrasound is available the mandatory pelvic radiograph of the infant in a Pavlik harness may not be necessary. ${ }^{30-32}$ Additionally, ultrasound may be able to detect the hips which do resolve spontaneously. ${ }^{33} \mathrm{~A}$ delayed examination of the hips at 2 weeks of age reduces the treatment rate and assists the assessment of hip location in those cases that do require treatment. ${ }^{34}$

\section{CONCLUSIONS}

Six cases of complications resulting from the use of splintage are presented. Complications may 
be prevented by observation of fundamental principles. The decision to splint an infant must not be undertaken lightly. A proportion of unstable hips do become normal by 2 weeks of age and splinting a normal hip may cause vascular injury. An infant placed in an abduction splint requires regular supervision by trained specialists to prevent any relapse of instability or complication resulting from the splint itself. Excessive abduction against tight hip adductors must be avoided particularly if rigid splintage is used. Diagnosis may be improved by ultrasound examination and the supervising specialist must also be aware that in certain cases splintage may have to be discontinued and alternative treatment such as adductor tenotomy or planned open reduction performed.

1 Pravas M. Du traitment de la luxation congenitale du femur. Bulletin de L'Academie de Medicine 1837;2:579.

2 Paci A. Nuovo contributo alla patologica della lussacione iliaca del femore. Archivo ed Atti della Societa Italiana di Chirugia 1887;3:444.

3 Severin E. Contribution to the knowledge of congenital dislocation of the hip joint. Late results of closed reduction and arthrographic studies of recent cases. Acta Chir Scand and arthrographic

4 Ponseti I. Causes of failure in the treatment of congenital dislocation of the hip. F Bone foint Surg [Am] 1944;26:775

5 Ilfield FW. Management of congenital dislocation and dysplasia of the hip by means of a special splint. Clin Orthop 1957;39A:99-110.

6 Browne D. The treatment of congenital dislocation of the hip Proceedings of the Royal Society of Medicine 1948;41:388-90.

7 Von Rosen S. Diagnosis and treatment of congenital dislocation of the hip in the newborn. $\mathcal{f}$ Bone foint Surg [Br] 1962;44:284-91.

8 Nicholson JT, Kopell HP, Mattei FA. Regional stress angiography of the hip: a preliminary report. $\mathcal{F}$ Bone foint Surg [Am] 1954;36:503-10.

9 Salter RB, Kostuic J, Dallas S. Avascular necrosis of the femoral head as a complication of treatment for congenital dislocation of the hip in young children; a clinical and dislocation of the hip in young children; a clinical a

10 Pavlik A. Die functionelle Behandlungsmethode mittels Riembenbugel als Prinzip der Konservativen Therapie bei angeborenen Huftgelenksverrenkungen der Saulinge. Zeitschrift fur Orthopaedics 1957;89:341-52.

11 Johnson AH, Aadalen RJ, Eilers VE, Winter RB. Treatment of congenital hip dislocation and dysplasia with the Pavlik harness. Clin Orthop 1981;155:25-9.

12 Ramsey PL, Lasser S, MacEwen GD. Congenital dislocation of the hip: use of the Pavlik harness in the child during the first six months of life. F Bone Foint Surg [Am] 1976;58: $1000-4$.
13 Iwasaki K. Treatment of congenital dislocation of the hip by the Pavlik harness; mechanism of reduction and usage. f Bone foint Surg [Am] 1983;65:760-7.

14 Suzuki $R$. Complications of the treatment of congenital dislocation of the hip by the Pavlik harness. Int Orthop dislocation of

15 Mubarak S, Garfin S, Vance R, McKinnon B, Sunderland D. Pitfalls in the use of the Pavlik harness for the treatment of congenital displasia, subluxation and dislocation of the hip f Bone Foint Surg [Am] 1981;63:1239-48.

16 Putti V. Early treatment of congenital dislocation of the hip. f Bone foint Surg [Am] 1929;11:798.

17 Weiner DS, Hoyt WA Jr, O'Dell HW. Congenital dislocation of the hip. The relationship of pre-manipulation traction and age to avascular necrosis of the femoral head. $\mathcal{F}$ Bone Foint Surg [Am] 1977;59:306-11.

18 Poole RD, Foster BK, Paterson DC. Avascular necrosis in congenital hip dislocation (significance of splintage). $\mathcal{F}$ Bone foint Surg $[\mathrm{Br}]$ 1986;68:427-30.

19 Kalamchi A, MacEwen GD. Avascular necrosis following treatment of congenital dislocation of the hip. $\mathcal{F}$ Bone foint treatment of congenital dis.

Surg [Am] 1980;62:876-8.
20 Barlow TG. Early diagnosis and treatment of congenital dislocation of the hip. $\mathcal{F}$ Bone foint Surg $[\mathrm{Br}]$ 1962;44: dislocatio

21 MacKenzie IG. Congenital dislocation of the hip; the development of a regional service. $\mathcal{F}$ Bone foint Surg [Br] 1972;54:18-39.

22 Mitchell G. Problems in the early diagnosis and management of congenital dislocation of the hip. $\mathcal{F}$ Bone foint Surg $[\mathrm{Br}]$ 1972;54:4-13.

23 Staheli LT, Dion M, Tuell JT. The effect of the inverted limbus on closed management of congenital hip dislocation. Clin Orthop 1978;137:163-6.

24 Gage JR, Winter RB. Avascular necrosis of the femoral epiphysis as a complication of closed reduction of congenital dislocation of the hip: a critical review of 20 years experience at the Gillette Children's Hospital. F Bone foint experience at the Gillette Child

25 Schwentker EP, Zaleski RJ, Skinner SR. Medial knee instability complicating the Pavlik harness treatment of instability complicating the Pavlik harness treatment of congenital hip

26 Renshaw TS. Inadequate reduction of congenital dislocation of the hip. F Bone foint Surg [Am] 1981;63:1114-21.

27 Almby B, Hjelmstedt A, Lonnerholm T. Neonatal hip instability - reason for failure of early abduction treatment Acta Orthop Scand 1979;50:315-27.

28 Scaglietti O, Calandriello $B$. Open reduction of congenita dislocation of the hip. $\mathcal{f}$ Bone foint Surg $[\mathrm{Br}] 1962 ; 44$ 257-83.

29 Somerville E, Scott J. The direct approach to congenital dislocation of the hip. 7 Bone foint Surg [Br] 1957;39:623.

30 Clarke NMP, Harcke HT, McHugh P, et al. Real time ultrasound in the diagnosis of congenital dislocation and dysplasia of the hip. F Bone foint Surg $[\mathrm{Br}]$ 1985;67: dysplasia

31 Jones DA, Powell N. Ultrasound and neonatal hip screening. A prospective study of 'high risk' babies. F Bone foint Surg [Br] 1990;72:457-9.

32 Terjesen T, Bredland T, Berg V. Ultrasound for hip assessment in the newborn. F Bone foint Surg [Br] 1989;71:767-73.

33 Clarke NMP. Sonographic clarification of the problems of neonatal hip instability. $\mathcal{F}$ Pediatr Orthop 1986;6:527-32.

34 Clarke NMP, Clegg J, Al-Chalabi AN. Ultrasound screening of hips at rest for CDH. Failure to reduce the incidence of late cases. F Bone foint Surg [Br] 1989;71:9-12. 